\title{
Editorial: Long-Memory Models in Mathematical Finance
}

\author{
Tommi Sottinen $^{1 *}$, Elisa Alòs ${ }^{2}$, Ehsan Azmoodeh ${ }^{3}$ and Giulia Di Nunno ${ }^{4}$ \\ ${ }^{1}$ School of Technology and Innovations, University of Vaasa, Vaasa, Finland, ${ }^{2}$ Department of Economics and Business, Pompeu \\ Fabra University, Barcelona, Spain, ${ }^{3}$ Deparment of Mathematical Sciences, University of Liverpool, Liverpool, United Kingdom, \\ ${ }^{4}$ Department of Mathematics, University of Oslo, Oslo, Norway
}

Keywords: mathematical finance, long-range dependence, long-memory, stochastic processes, stochastic analysis, heavy tails

\section{Editorial on the Research Topic}

\section{Long-Memory Models in Mathematical Finance}

Long-memory models are statistical models that describe strong correlation or dependence across time-series data. These are typically characterized by an autocovariance that is decaying slowly with the increase of the lag between observations. This is often taken in comparison with the classical autoregressive moving average (ARMA) time-series, where the autocorrelation decays exponentially fast resulting a fairly negligible correlation between distant times.

Evidence of long-range dependence was detected in various fields of natural and social sciences. In this volume, our interests focus on the analysis of time-series from finance. In this setting evidence of long memory has been found not quite so much at the level of the raw time series, but in the analysis of their squares (early works include, e.g., Refs. 1 and 2). The impact of this findings is evident in the volatility modeling of asset prices, since then developed.

The analysis of stationary time-series is crucial in finance. Prices and volatilities do not follow classical diffusion models, but their structure is much more complicated. In this framework, central limit theorems play an important role. Recently, the combination of Stein's method and Malliavin calculus has given new tools for the study of central limit theorems for Gaussian subordinated stationary processes (processes of the form $f(X)$, where $f$ is a function and $X$ is a Gaussian process). In the first contribution [Viitasaari-Ilmonen], Lauri Viitasaari and Pauliina Ilmonen focus on the study of weakly stationary time-series. More precisely, the authors prove that these processes can be viewed as Gaussian subordinated processes, in the sense that both processes have the same marginal distributions and, asymptotically, the same covariance function, and they provide provide limiting limits for the standard mean and autocovariance estimators. This approach has some advantages, being more flexible and needing weaker hypotheses as, for example, linear approximations.

In the second contribution [Voutilainen], Marko Voutilainen introduces an autoregressive model of order 1 type characterization covering all multivariate strictly stationary processes indexed by the set of integers. Under the adequate square integrability assumption, he deduces continuous-time algebraic Riccati equations for the parameter matrix. This enables him to naturally define the associated estimator and show that the estimator inherits consistency from autocovariances of the stationary process. The limiting distribution can be further realized as a linear function of the limiting distribution of the autocovariances.

Volatility models based on the fractional Brownian motion $(\mathrm{fBm})$ are of great interest in the modeling of the long-term and short-term implied volatility surface. As $\mathrm{fBm}$ volatilities are not Markovian, their numerical analysis is complicated. In this scenario, one challenging problem is calibration. In the third contribution [Garnier-Sølna], Josselin Garnier and Knut Sølna focus on this problem. In a set of scaling regimes, the authors derive asymptotic approximations for option prices 
and implied volatilities. These expansions can be written in terms of effective parameters, that can be easily estimated from real market data and provide a robust calibration. This methodology gives a new tool in the calibration of volatility models that exhibit long or short memory properties.

In the fourth contribution [Lavagnini], Silvia Lavagnini considers approximation of continuous-time autoregressive moving-average processes (CARMA, for short). These are continuous-time analogs of the moving-average processes and they are defined as solutions of stochastic differential equations (SDE) with a Lévy process as the driving noise. The CARMA processes are widely used to model heavy tails which are, while not a long-range dependence property, closely related in the sense that both heavy tails and long-range dependence can manifest similar scaling laws. Heavy tails are frequently observed in

\section{REFERENCES}

1. Ding Z, Granger CWJ, and Engle RF. A Long Memory Property of Stock Market Returns and a New Model. J Empirical Finance (1993). 1:83-106. doi:10.1016/ 0927-5398(93)90006-D

2. Robinson PM. The Memory of Stochastic Volatility Models. J Econom (2001). 101:195-218. doi:10.1016/S0304-4076(00)00079-8

3. Brockwell PJ. Lévy-Driven Carma Processes. Ann Inst Stat Math (2001). 53: 113-24. doi:10.1023/A:1017972605872

4. Svetlozar T. Rachev: Handbook of Heavy Tailed Distributions in Finance. NorthHolland (2003). doi:10.1016/B978-0-444-50896-6.50021-2 financial time-series, see Refs. 3 and 4 . Consequently, the CARMA processes have recently gained popularity in econometric modeling. In her paper Lavagini considers approximations of the CARMA processes, where the driving Lévy process is replaced by a smooth process. Her approximation justifies numerical Euler schemes for simulation of CARMA processes. The approximation error bounds and corresponding Euler schemes are analyzed carefully. Simulation and parameter estimation results are given.

\section{AUTHOR CONTRIBUTIONS}

All authors listed have made a substantial, direct, and intellectual contribution to the work and approved it for publication.

Conflict of Interest: The authors declare that the research was conducted in the absence of any commercial or financial relationships that could be construed as a potential conflict of interest.

Copyright (C) 2021 Sottinen, Alòs, Azmoodeh and Di Nunno. This is an open-access article distributed under the terms of the Creative Commons Attribution License (CC $B Y)$. The use, distribution or reproduction in other forums is permitted, provided the original author(s) and the copyright owner(s) are credited and that the original publication in this journal is cited, in accordance with accepted academic practice. No use, distribution or reproduction is permitted which does not comply with these terms. 